\title{
Incidence of Methicillin Resistant Staphylococcus aureus Infection in Neonatal Septicemia in a Tertiary Care Hospital
}

\author{
S. Rajesh*, B. Divya, D. Neelaveni and N. Subathra \\ Department of Microbiology, Government Mohan Kumaramangalam Medical College, \\ Salem, Tamil Nadu, India \\ *Corresponding author:
}

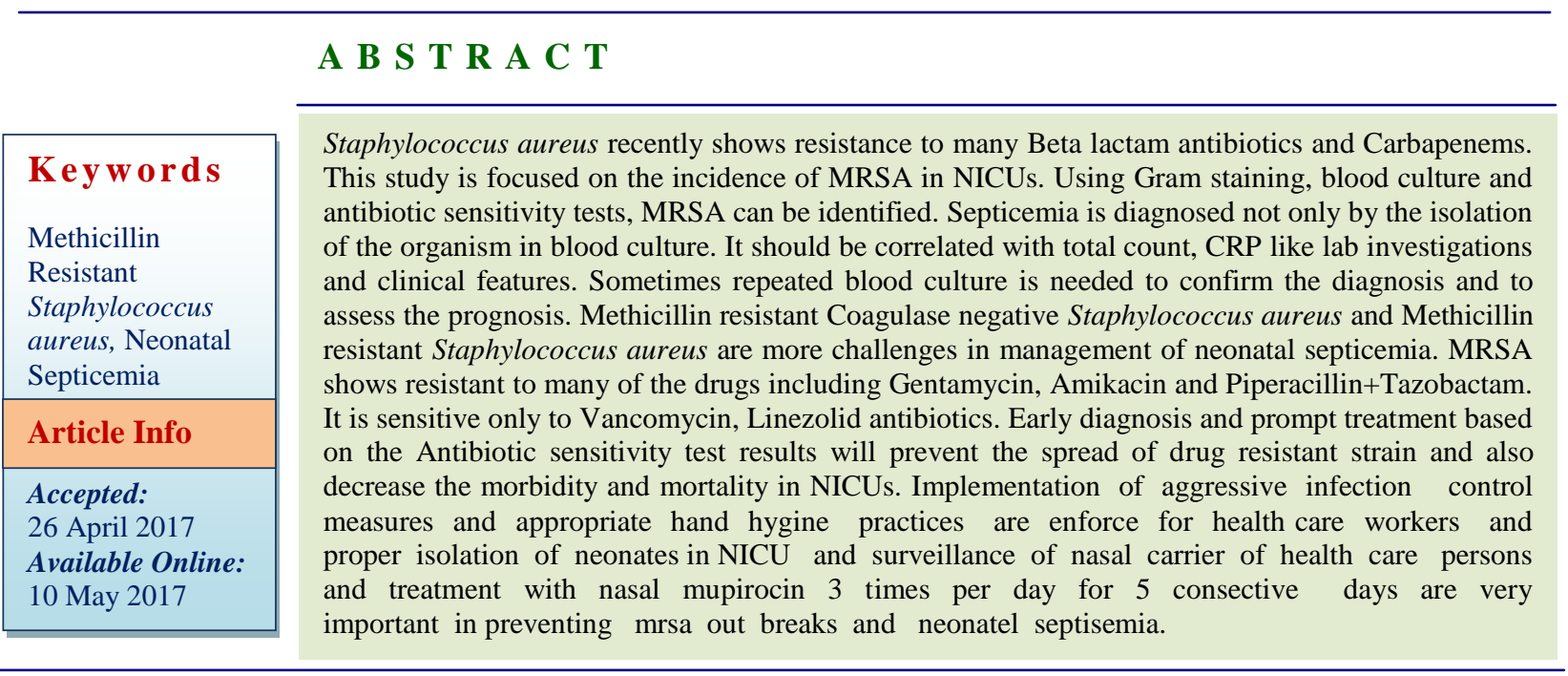

\section{Introduction}

Methicillin resistant Staphylococcus aureus is a frequent source of infections affecting premature and critically ill infants in Neonatal Intensive Care Unit. MRSA was first isolated in hospitals in the united kingdom in 1961. The proportion of healthcare associated Staphylococcal infection in united states intensive care unit due to MRSA has continued to rise from $35.9 \%$ in 1992 to $64.4 \%$ in 2003. 20years after the first MRSA case was described, the first neonatal case of MRSA infections occurring in a neonatal intensive care unit (NICU) was published. Since that time MRSA has become a frequent source of infections affecting premature and critically ill neonates in NICUs. It is a bacterium responsible for various skin and soft tissue infections in neonates. Staphylococcus aureus was found to be responsible for $7.8 \%$ of cases of late onset sepsis and $3 \%$ of cases of early onset sepsis in very low birth weight neonatal infants. Furthermore Methicillin resistant Staphylococcus aureus, either nosocomially or community acquired, has emerged as a challenging pathogen in NICUs.

Long before, Penicillins had been the drug of choice for treating Staphylococcus aureus infections. But, unfortunately excessive use of those Penicillins over the years has led to the development of stronger strains of 
Staphylococcus aureus that are no longer killed by Penicillin antibiotics.

MRSA is any strain of Staphylococcus aureus that has developed, through horizontal gene transfer and natural selection, multiresistance to Beta lactam antibiotics, which include the Penicillins (Methicillin, Dicloxacillin, Nafcillin, Oxacillin, etc) and Cephalosporins. The evolution of such resistance does not cause the organism to be more intrinsically virulent than strains of Staphylococcus aureus that has no antibiotic resistance, but resistance does make MRSA infection more difficult to treat with standard types of antibiotics and thus are more dangerous. MRSA can be Hospital acquired, Community acquired and Livestock acquired. This study focuses on Hospital acquired MRSA.

Methicillin resistance is mediated by the mecA gene, which encodes for a novel Penicillin binding protein, PBP-2a. In MRSA, exposure to Methicillin inactivates the 4 high binding affinity PBPs normally present.PBP$2 \mathrm{a}$ which displays a low affinity for Methicillin, takes over the function of these PBPs, permitting the cell to grow. Two genes located upstream from mecA-mecR1 and mec1 control expression of PBP2a.

Tertiary care medical college hospitals are major reference centre and receiving critically ill and sepsis suspected neonates from private hospitals and government primary health centres. sepsis is a major cause of neonatel mortality in developing countries. early onset of sepsis (eos) was defined as positive blood culture within 72 hours of life (birth) and late onset sepsis was defined positive blood culture after 72 hours of life. neonatal septisemia was defined as significant organism either bacterial (or) fungal isolated on blood culture with onr (or) more clinical features (or) with laboratory features of sepsis. clinical features of sepsis included: 1.fever 2. lethargy 3. respiratory distress 4.apnoea 5. cynosis 6.abdominal distension 7 . bradycardia 8 . impired perfusion. Laboratory features of : reduced platelet, increased WBC count, ESR and $\mathrm{C}$ reative protein.

Early onset of sepsis usually related to peripartum factors before (or)during delivery. while late onset sepsis (los) acquired in newborn nursery. in developed countries group b strepto cocci is important causative organism in early neonatal sepsis . in developing countries cons, e.coli, klebsiella are commenest organisms. Maternal risk factors identified were preterm labour, PROM and intrapartum fever. Swelling and tenderness of the affected body part. Here are some examples of invasive infection which were noticed in the neonates.

Being responsible for these life threatening conditions, MRSA has emerged as a challenging pathogen in NICUs, with high mortality and morbidity rates. This study has been undertaken to prove the increasing incidence of the challenging MRSA. This study insists on the severe necessity for continued surveillance of MRSA. It also dispels the common notion among practitioners that Gram negative bacteria are the predominant isolates in neonatal septicemia.

Staphylococcus aureus is responsible for $7.8 \%$ cases of late onset sepsis and 3\% cases of early onset sepsis [1]. MRSA can be Hospital acquired, Community acquired and Livestock acquired [3]. There is a recent predominance of Gram positive organisms in neonatal sepsis [4]. KIRBY BAEUR DISC DIFFUSION method is the most reliable one for antibiotic sensitivity testing [6]. Criteria for Cefoxitin disc diffusion test (Diameter of zone of inhibition < 22mm - MRSA / 
Diameter of zone of inhibition > $22 \mathrm{~mm}$ MSSA) [7].

The main aim of this study, to find out whether the Staphylococcus aureus isolated from the neonates were Methicillin resistant by appropriate techniques. And to study the incidence and outcome of systemic infections with MRSA infections. Also to understand the recent predominancy of Gram positive organisms in causing neonatal septicemia.

\section{Materials and Methods}

This is a prospective study undertaken in the department of Diagnostic Microbiology, Govt. Mohan Kumaramangalam medical college hospital, SALEM. A minimum of 110 neonates hospitalized in the NICU were included. Blood sample was collected from the neonates for a period of 6 months excluding all patients above one month.

\section{Methodology}

\section{Sample collection}

$1 \mathrm{ml}$ of peripheral blood was collected aseptically and placed in an aerobic bottle with $10 \mathrm{ml}$ of Brain Heart Infusion broth.

\section{Transport}

Blood collected from the infant was immediately taken to the Microbiology department in Brain Heart Infusion broth blood culture bottles.

\section{Gram Staining}

Gram staining of the blood samples showed Gram positive cocci in grape like clusters.

\section{Culture}

Blood was incubated for 3 to 7 days and subculture was done on Blood agar, MacConkey agar and Mannitol salt agar. The organism was confirmed as Staphylococcus aureus by the formation of white opaque colonies with beta hemolysis in Blood agar, yellow colonies in Mannitol salt agar and tiny pink colonies in MacConkey agar.

\section{Biochemical reactions}

Various biochemical reactions were performed. The organism showed the following results. Catalase positive, Indole negative, Methyl red positive, VP positive, Urease positive, Coagulase positive.

The culture was confirmed positive for Staphylococcus aureus by Gram staining of the colonies and by tube coagulase test.

\section{Antibiotic sensitivity test}

The Staphylococcal isolates from culture were subjected to testing for Methicillin resistance by KIRBY BAUER DISC DIFFUSION method. This method makes use of MuellerHinton agar plate. Using a sterile swab, the cultured bacteria $\left(1-2 \times 10^{8} \mathrm{CFU} / \mathrm{ml}\right)$ is inoculated to the surface of the MuellerHinton agar plate. The $\mathrm{pH}$ of the agar is maintained between 7.2-7.4. The plate is dried for 5 minutes. Using an antibiotic disc dispenser, Cefoxitin is placed on to the plate. The plate is then incubated at $37^{\circ} \mathrm{C}$ for $48 \mathrm{hrs}$. The susceptibility to Methicillin is measured by the diameter of the zone of inhibition.

Oxacillin can also be used for this method but Cefoxitin is an even better inducer of mecA gene and tests using Cefoxitin give more reproducible and accurate results.

The following antibiotics were tested for sensitivity. Cefoxitin, Amoxicillin, Cefotaxime, Erythromycin, Gentamycin, Amikacin, Ciprofloxacin, Piperacillin+ Tazobactam, Vancomycin, Linezolid 


\section{Results and Discussion}

Among the 400 specimens was received from neonatal intensive unit during study period, 8 are identified as contaminates and not included in significant isolate (bacillus, micrococcus, corny bacterium). Out of 400 cases which were included in our study with suspected for sepsis, 68 cases yielded positive results for growth of pathogen in blood culture. Only 68 of blood culture samples are shows significant positive for microbial cultures. All these positive blood culture growth occurred within 48 hours to 72 hours subculture. No growth occurred after 72 hours subculture.

Among 68 septic neonates 30 neonates (44 $\%)$ are presented as early onset sepsis and CONS, MRSA, E.coli, are present as main isolation. 38 neonates are presented as late onset sepsis (56\%) and Enterococcus, MRSA and Klebsiella and CONS are present as main isolation. Among these isolates, Staphylococcus was the predominant organism (41 out of 68). Staphylococcus aureus were 14 which included 8 MRSA and 6 MSSA. Coagulase negative Staphylococcus were 27 which included 16 MR-CoNS and 11 Ms-CoNS.

Among 27 coagulase negative staphylococcus 23 are Staphylococcus epidermidis and 4 are other species of CONS. 8 methicilline resistant Staphylococcus aureus and 16 methicilline resistant coagulase negative staphylococcus and totally 24 Staphyloccal isolates were Methicillin resistant.

Other organisms were Klebsiella, Escherichia coli, Pseudomonas, Acinetobacter and Enterococci.

Table. 1

\begin{tabular}{|c|c|c|}
\hline Name of organism & Number of isolations & X $\%$ \\
\hline \multicolumn{3}{|c|}{ GPC } \\
\hline MRSA & 08 & $11.7 \%$ \\
\hline MSSA & 06 & $8.8 \%$ \\
\hline MR-CoNS & 16 & $23.5 \%$ \\
\hline MS-CoNS & 11 & $16.2 \%$ \\
\hline Enterococci & 05 & $7.35 \%$ \\
\hline Gpc total & 46 & $67.6 \%$ \\
\hline \multicolumn{3}{|c|}{ GNB } \\
\hline Klebsiella & 11 & $16.2 \%$ \\
\hline Escherichia Coli & 07 & $10.3 \%$ \\
\hline Pseudomonas & 01 & $1.47 \%$ \\
\hline Acinetobacter & 03 & $4.4 \%$ \\
\hline Gnb total & 22 & $32.4 \%$ \\
\hline
\end{tabular}


During the study period of 6 months in the NICU, a clear idea of the clinical course of MRSA infection was obtained. These MRSA were isolated from the neonates who had many predisposing factors and are the following;

Poorly developed host defence mechanisms. Central venous catheterization; Endotracheal and upper GIT tube replacement procedures causing interruption in skin integrity; A prolonged parenteral nutrition; Use of steroids, antimicrobial agents.

This clearly shows that MRSA isolated from the neonates in NICU were mainly hospital acquired. Staphylococcal infection may occur by surface contamination and also from healthcare workers. Doctors and laboratory workers sometimes act as carriers. Mupirocin is used for the treatment of nasal carriers. Screening of nasal carriers and appropriate treatment is important to prevent spreading of outbreaks. Regular screening of NICU for aerobic organism by open plate culture method is very important to prevent neonatal septicemia. Coagulase negative Staphylococcus aureus is a normal skin flora. It becomes pathogenic in a immunocompromised individual. Also repeated venous puncture for blood collection leads to invasion of the organism in to the blood. This insists upon the strict aseptic precautions before clinical procedures such as hand washing techniques, Sterilization of equipments, isolation of the colonized neonate, etc. Continued surveillance for MRSA and other emerging multidrug resistant pathogen becomes very important. From antibiotic sensitivity patterns, it was clear that the organism was resistant to many of the antibiotics and was sensitive only to Vancomycin and Linezolid.

Out of 68 neonates males were $36(53 \%)$ and females were $32(47 \%)$. It is similar to Sartaj bhat et al study. In the present study total of 68 isolation from blood culture $67.6 \%$ are gram positive cocci and only $32.4 \%$ are gram negative bacilli. In 400 blood culture only 68 shows positive isolation and incidence neonatal sepsis was $17 \%$ in neonatal unit during the our study period which is less as compared to other study like Shashi Gandhi et al shows $32 \%$ and positivity rate of $28 \%$. In Urvashi Rana at al study, both EOS \& LOS are almost equal in present study. $44 \%$ are presented as early onset sepsis and (56\%) presented as late onset sepsis but Premalatha et al study shows $64 \%$ neonatel sepsis presented with early onset sepsis and Sudharshan Raj, Shipragal hotra et al., Study shows $83 \%$ was early onset sepsis and $17 \%$ late onset sepsis.

The present study shows $39.7 \%$ Coagulase negative staphylo coccus and $20.6 \%$ Staphylococcus aureus and in total $60.3 \%$ are the predominantly Staphylococcus pathogen. But study like (1) Shashi Gandhi et al shows only $35 \%$ gpc and more number of 65\% gnb and in Abhishek M Mehta et al (2) study showed that Staphylococcus aureus was the most predominant isolate $(54.6 \%)$.

In our study maximum number of $39.7 \%$ coagulase negative Staphylococcus and Sunder et al (14) bhdulaziz s. Et al (15) Study also shows predominant isolates are coagulase negative staphylococcus as similar to our study.

The present study only $20.6 \%$ isolates are staphylococcus aureus. Shaw CK et al study shows The most common organism to be isolated was staphylococcus aureus (42.75\%) followed by Klebsiella pneumoniae (18.32\%) Shipragal hotra et al (16) Study shows predominant isolates $32 \%$ are Staphylococcus aureus. Urvashi Rana at al study shows more number of group b streptococcus $7 \%$ and Enterococcus $12 \%$ like gram positive 
organisms. But our study only $4.4 \%$ of Enterococcus isolated.

Group B Streptococcus, a common cause of neonatal sepsis in the Western countries is infrequent in India and in other tropical countries. In the present study also shows the absence of group b streptococcus organism causing sepsis in newborn.

Even GPC isolated more in number, associated with less mortality but gnb isolated in less number associate with more mortality.

In the study less number of gram negative bacilli $32.4 \%$ isolated which include $16 \%$ Klebsiella and $10 \%$ of e.coli. Urvashi Rana at al (5) study shows $75 \%$ of gnb and Shasi Gandhi et al (1)study shows more number of $60 \%$ gnb and mainly $41 \%$ of E.coli and in (6) Sartaj bhat et al study shows Klebsiella sepsis was most common $40 \%$, followed by Acinetobacter (15\%), E. coli (7.5\%) . And Hura Kanwaljeet Singh et al(12) shows Klebsiella pneumoniae was the most common isolate accounting for $49.64 \%$ cases followed by Escherichia coli $26.95 \%$, Pseudomonas aeruginosa $7.80 \%$,

In our study only one pseudomonas is isolated. Begum Sharifun Naher et al study shows Gram negative organisms were $(78 \%)$ and Pseudomonas aeroginosa 27 (46.55\%).

In the present study shows no fungus isolated. But Sartaj bhat et al(6) study shows $6.25 \%$ candida fungus as isolation.

In the present study $65 \%$ of Methicilline sensitive Staphylococcus aureus and Methicillin sensitive coagulase negative Staphylococcus are response to cefotaxime or ampicilline, amoxyclave, gentamycin and amikacin like antibiotics. Even $40 \%$ of Methicillin resistant Staphylococcus aureus and Methicillin resistant coagulase negative
Staphylococcus are response to cefotaxime, ampicilline, amoxyclave, gentamycin and amikacin like antibiotics. Premalatha et al study shows gram positive staphylococcus aureus were ampicillin resistant about $87 \%$ and CONS was resistant to ampicilline about 76\%. And more than $70 \%$ Resistant to gentamycin, amoxy-clav. 50\% of the isolates were sensitive to ciprofloxacin.

Also Hura Kanwaljeet Singh et al(12) study shows more number of resistance was found against various antibiotics such as ampicillin (73\%), Third generation cephalosporins (61\%) and combination drugs such as piperacillin-tazobactum (13\%). MR-CONS shows more resistance to ampicillin and cefotaxime and erythromycin.

Linzolid and vancomycin restricted to use only for culture proven methicillin resistant staphylococcus sepsis.

In conclusion, a gradual increase in the ratio of MRSA colonization and infections in a tertiary care hospital was identified and the importance of abiding by strict infection control policies, including hand hygiene and proper isolation practices was recognized.

It is also understood that Gram positive organisms have recently emerged as predominant cause of neonatal septicemia. There has been a shift from the predominance of Gram negative organisms to Gram positive organism Staphylococcus aureus. Implementation of aggressive infection control measures and appropriate hand hygiene practices are enforce for health care workers and proper isolation of neonates in NICU and surveillance of nasal carrier of health care persons and treatment with nasal mupirocin 3 times per day for 5 consective days are very important in preventing mrsa out breaks and neonatel septicemia 


\section{References}

1. Shashi Gandhi et al. Neonatal Sepsis in Tertiary Care Hospital an overview more $60 \%$ gnb $21 \%$ e.coli. International Journal of Medical Science and Public Health | 2013 | Vol 2: Issue 3.

2. Abhishek M Mehta, Navinchandra $M$. Kaore. Research Article Microbial Profile of Neonatal septicaemia in a tertiary care hospital of Bhopal. International Journal of Biomedical And Advance Research.

3. Sudharshan Raj. C, Dr. Pradeep Reddy. M2, Dr. Neelima. A. BACTERIOLOGICAL PROFILE OF NEONATAL SEPSIS IN ATERTIARY CARE HOSPITAL. WORLD JOURNAL OF PHARMACY AND PHARMACEUTICAL SCIENCES. Volume 2, Issue 6, 5709-5717. Research Article ISSN 2278 - 4357

4. International Journal of Recent Trends in Science And Technology, ISSN 22772812 E-ISSN 2249-8109, Volume 10, Issue 3 2014, The Bacterial Profile and Antibiogram of Neonatal Septicaemia in a Tertiary Care Hospital, D. E. Premalatha1*, Mallikarjun Koppad2, L. H. Halesh3, K.C. Siddesh4,

5. Urvashi Rana. Clinico-Bacteriological Profile of Neonatal Sepsis in a Tertiary Care Hospital. ARC Journal of Pediatrics Volume 2, Issue 2, 2016

6. The Clinical and Bacteriogical Spectrum of Neonatal Sepsis in a Tertiary Care Hospital, Deen Dayal Upadhyay Hospital, Harinagar New Delhi India 1Sartaj Bhat, 2Suhail Naik, 3Musadiq Alaqaband IOSR Journal of Dental and Medical Sciences .Volume 14, Issue 6

7. BANGLADESH J CHILD HEALTH 2013; VOL 37 (1) : 14-17Neonatal Sepsis in A Tertiary Care Hospital: Evaluation of

Causative Agents and Antimicrobial
Susceptibilities Begum Sharifun Naher, Syeda Afroza

8. Kathmandu University Medical Journal (2007), Vol. 5, No. 2, Issue 18, 153-160 Neonatal sepsis bacterial isolates and antibiotic susceptibility patterns at a NICU in a tertiary care hospital in western Nepal: A retrospective analysis Shaw CK1, Shaw P1, Thapalial A3

9. Sanjay D Rathod1, Palak V Bhatia2, NEONATAL SEPTICEMIA AT TERTIARY CARE HOSPITAL OF AHMEDABAD NATIONAL JOURNAL OF MEDICAL RESEARCH , Volume 2| Issue 4|Oct - Dec 2012

10. AETIOLOGY AND PRESENTATION OF NEONATAL SEPTICAEMIA AT TERTIARY CARE HOSPITAL OF SOUTHERN RAJASTHAN

Dr.Deepandra Garg1*, International Journal of Medical Science and Education

11. International Journal of Medical Research Health Sciences

Volume 4 Issue 3

BACTERIOLOGICAL PROFILE OF NEONATAL SEPTICEMIA: A RETROSPECTIVE ANALYSIS

FROM A TERTIARY CARE HOSPITAL IN LONI

*Sneha Ann Oommen1, Santosh Saini2

12. ejpmr, 2016,3(3), 241-245

BACTERIOLOGICAL PROFILE OF NEONATAL SEPSIS IN NEONATAL INTENSIVE CARE UNIT (NICU) IN A TERTIARY CARE HOSPITAL: PREVALENT BUGS AND THEIR SUSCEPTIBILITY PATTERNS

Hura Kanwaljeet Singh, MD, 2Phuljhele Sharja

13. Eastern Journal of Medicine 17 (2012) 119-125The clinical spectrum and outcome of neonatal sepsis in a neonatal intensive care unit at a tertiary care 
hospital Chandan Kumar Shawa, ${ }^{*}$, cons neonatal sepsis in tertiary care hospital, ijcmas,sunder et al vol5,2016

15. neonatal sepsis in tertiary care hospital at Saudi ,bhdulaziz s. et al, international j.of advanced research,cons overall and e.coli 60 los

16. clinico bacterio profile of neonatal sepsis ,shipragalhotra et al ,journal of mahatma Gandhi inistitute of medical science 2015, vol 20,issue 2 eos 82 and los 17 gpc s.aureus.

\section{How to cite this article:}

Rajesh, S., Divya, Neelaveni and Subathra. 2017. Incidence of Methicillin Resistant Staphylococcus aureus Infection in Neonatal Septicemia in a Tertiary Care Hospital. Int.J.Curr.Microbiol.App.Sci. 6(5): 2874-2881. doi: http://dx.doi.org/10.20546/ijcmas.2017.605.327 